\title{
Uso concomitante de benzodiacepinas e hipoglucemiantes orales en pacientes diabéticos tipo 2
}

\author{
Agostina Fornero, Pamela Bertoldo, Nilda Dumont \\ Facultad de Ciencias Químicas. Secretaría de Investigación. Universidad Católica de Córdoba. Argentina.
}

\section{PALABRAS CLAVE}

Benzodiacepinas, diabetes mellitus tipo 2, glucemia preprandial, hipoglucemiantes orales

\section{ABREVIATURAS}

BZD: benzodiacepinas DM: diabetes mellitus DM2: diabetes mellitus tipo 2 GABA: ácido gama amino butírico

HGO: hipoglucemiantes orales

\section{KEYWORDS}

Benzodiazepines, diabetes mellitus type 2, pre-meal blood sugar level, oral glucose-lowering drugs

\section{RESUMEN}

Introducción: En los pacientes diabéticos en tratamiento con hipoglucemiantes orales (HGO) se observan disminuciones de las glucemias preprandiales mayores de las esperadas.

Objetivo: Evaluar en pacientes diabéticos tipo 2, con hipoglucemiantes orales, la relación entre el efecto producido por el consumo de benzodiacepinas y el nivel de glucosa preprandial en el autocontrol realizado por el paciente e hipoglucemias.

Métodos: Se realizó un estudio de tipo observacional, descriptivo, transversal realizado en una farmacia comunitaria de la ciudad de Córdoba (Argentina). Se utilizaron promedios de las glucemias preprandiales. Se dividen en dos grupos: con o sin prescripción conjunta de benzodiacepinas. Se compararon los grupos utilizando prueba T de Student.

Resultados: Metformina fue el HGO más prescrito $(n=42)$ como monoterapia o asociación, combinándose con más frecuencia con alprazolam $(n=9)$ y diazepam $(n=5)$. Las benzodiacepinas más prescritas fueron alprazolam (15 pacientes) y diazepam (13 pacientes). Diazepam ha sido la benzodiacepina que provoca mayor descenso de la glucemia $(n=8)$ seguido de alprazolam $(\mathrm{n}=7)$. En el grupo que tiene prescripción de ambos medicamentos existe una disminución de los niveles de glucemias preprandiales llegando en 15 (35\%) pacientes a niveles de hipoglucemia (menor a $70 \mathrm{mg} / \mathrm{dl}$ ).

Conclusiones: La utilización de benzodiacepinas junto con HGO en este grupo de pacientes diabéticos tipo 2 logró un mejor control de los niveles de glucemia preprandial que en aquellos que sólo emplearon monoterapia de HGO. Sin embargo, debido a las limitaciones metodológicas del estudio no se puede concluir una relación directa.

Concomitant use of benzodiazepines and oral glucose-lowering drugs in patients with type-2 diabetes

\section{ABSTRACT}

Introduction: In diabetic patients undergoing treatment with oral glucose-lowering drugs (GLD), greater-than-expected decreases were observed in pre-meal blood sugar levels.

Objective: In type 2 diabetic patients taking oral glucose-lowering drugs, evaluate the relationship between the effect of taking benzodiazepines and the patient's self-monitored pre-meal glucose level in relation to hypoglycemia.

Methods: A cross-sectional, descriptive, observational study was conducted in a community pharmacy in the city of Córdoba (Argentina). Averages of pre-meal blood sugar levels were used. Subjects were divided into two groups: with and without concomitant prescription of benzodiazepines. The groups were compared using Student's t-test.

Results: Metformin was the most prescribed oral GLD $(\mathrm{n}=42)$ as a monotherapy or adjunctive therapy, most frequently combined with alprazolam $(n=9)$ and diazepam $(n=5)$. The most frequently prescribed benzodiazepines were alprazolam (15 patients) and diazepam (13 patients). Diazepam was the benzodiazepine that most effectively lowered the glucose level $(n=8)$, followed by alprazolam $(n=7)$. In the group where both drugs were prescribed, there is a decrease to hypoglycemic levels (less than $70 \mathrm{mg} / \mathrm{dl}$ ) in the pre-meal blood sugar levels of 15 (35\%) patients.

Conclusions: The use of benzodiazepines together with oral GLD in this group of type 2 diabetic patients resulted in better control of the pre-meal glucose levels than in the group for which only GLD monotherapy was used. However, due to the methodological limitations of the study, a direct link cannot be concluded.

Esta investigación ha sido realizada como parte del trabajo final de la carrera de Farmacia de Agostina Fornero.

Recibido: $16 / 12 / 2017$

Aceptado: 24/2/2018

Disponible online: $30-3-2018$
Financiación: Secretaria de Investigación de la Universidad Católica de Córdoba. Argentina. Conflicto de intereses: ninguno.

Contribución a la autoría: las tres autoras declaran haber contribuido por igual al diseño y desarrollo del estudio, el análisis de los resultados y la redacción y revisión del manuscrito.

Cite este artículo como: Fornero A, Bertoldo P, Dumont N. Uso concomitante de benzodiacepinas e hipoglucemiantes orales en pacientes diabéticos tipo 2. Farmacéuticos Comunitarios. 2018 Mar 30; 10(1):41-46. doi:10.5672/FC.2173-9218.(2018/ Vol10).001.06

Correspondencia: Pamela Bertoldo (pamela.bertoldo@gmail.com)

ISSN 1885-8619 @SEFAC (Sociedad Española de Farmacia Familiar y Comunitaria). Todos los derechos reservados. 


\section{Introducción}

La diabetes mellitus (DM) es un trastorno metabólico que se caracteriza por presentar hiperglucemia crónica y alteraciones del metabolismo de glúcidos, lípidos y proteínas [1]. Su causa se debe a una disminución en la secreción de la hormona pancreática insulina, una deficiencia en su acción o un déficit de utilización por parte del organismo; en algunos pacientes puede estar relacionada con factores genéticos [2]. Representa un problema en salud pública y es una de las enfermedades crónicas no transmisibles donde hay que intervenir preventivamente a fin de mejorar la calidad de vida de las personas enfermas.

Con el transcurso del tiempo se ha observado un aumento significativo de personas con DM, en 1980 se registraban 180 millones de pacientes, según la Organización Mundial de la Salud. Actualmente en el mundo hay aproximadamente 422 millones de personas padeciendo esta enfermedad. Esta patología es, en un 80\% de casos, causa de muerte de países con ingresos bajos y medios. Se espera para el año 2030 el doble de mortalidad registrada entre los años 2005-2015 y que se convierta en la séptima causa de muerte a nivel mundial. En Argentina representa el 3\% de las causas de muerte [3].

El objetivo global del tratamiento del paciente con DM es reducir las concentraciones sanguíneas de glucosa, llegando a valores normales que permitan mejorar signos, síntomas y prevenir o posponer la aparición de complicaciones [4]. Se consideran pacientes controlados aquellos con valores de glucemias preprandial entre $70-130 \mathrm{mg} / \mathrm{dl}$ y postprandial $<180$ $\mathrm{mg} / \mathrm{dl}$ (60 a 120 minutos posterior a la ingesta) [5]. El tratamiento propuesto por el Ministerio de Salud de la Nación en Argentina consiste en tener en cuenta cinco pilares fundamentales: plan de alimentación saludable, ejercicio físico, automonitorización de la glucemia, tratamiento farmacológico (hipoglucemiantes orales y/o insulina), educación diabetológica [6].

El control de la DM es un proceso diario y riguroso. Representa para muchos pacientes un reto importante y continuo, lo que puede desencadenar complicaciones por el impacto del estrés que predispone al aumento de las glucemias. En este tipo de pacientes se pueden encontrar prescripciones de benzodiacepinas (BZD), ade- más de sus hipoglucemiantes orales (HG0) e insulina, debido a que este grupo de fármacos presentan propiedades ansiolíticas, hipnóticas (además de las anticonvulsivantes y relajantes musculares) en función de la dosis prescrita, su vida media plasmática $\mathrm{y}$ metabolismo.

Las benzodiacepinas tienen un mecanismo de acción común, se unen al receptor $\mathrm{GABA}_{\mathrm{A}}$, generando un cambio conformacional que incrementa la acción inhibitoria del neurotransmisor GABA, mediador químico de la inhibición tanto en el nivel del botón presináptico como pos sináptico, en todas las regiones del sistema nervioso central [6].

Los cambios en la concentración de glucosa pueden modificar la liberación de neurotransmisores cerebrales. El GABA parece regular los niveles de insulina en el organismo; actuando como molécula de señalización extracelular en los islotes pancreáticos, ya que se ha demostrado la existencia de GABA en el páncreas. La liberación de GABA depende de las concentraciones de glucosa en sangre y se lleva a cabo con flujo de $\mathrm{Ca}_{2}^{+}$en canales dependientes de voltaje. Una vez que es liberado actúa de modo autócrino y parácrino sobre las células de los islotes para modular la secreción hormonal mediante la activación de los receptores $\mathrm{GABA}_{\mathrm{A}} \mathrm{y}$ $\mathrm{GABA}_{\mathrm{B}} \mathrm{y}$ así regular la liberación de insulina y glucagón. Este sistema mediado por el GABA en los islotes pancreáticos parece ser importante para mantener la homeostasis de la glucosa. El GABA actúa sinérgicamente con la insulina [7].

El objetivo de este trabajo, ante la falta de estudios recientes, es describir en pacientes diabéticos tipo 2 (DM2) no insulinizados, tratados con hipoglucemiantes orales, la relación entre el efecto producido por el consumo de benzodiacepinas y el nivel de glucosa preprandial y la presencia de hipoglucemias en el autocontrol realizado por el paciente.

\section{Materiales y métodos}

Se realizó un estudio de tipo observacional, descriptivo, transversal. Se seleccionaron pacientes de ambos sexos con DM2 que asistieron a una farmacia del centro de la ciudad de Córdoba, entre los meses de octubre y noviembre del año 2015.

\section{Sujetos}

Criterios de inclusión: diagnóstico médico de DM2 con edad igual o mayor de 40 años y prescripción de HGO, que retiraron tiras reactivas y conservaban registro de sus mediciones de glucosa en sangre de al menos 25 días con un mínimo de una medición diaria.

Criterios de exclusión: personas con diagnóstico médico de DM2 que utilizaban insulina y/o esquema mixto con menos de 25 dias de mediciones de autocontrol en el tiempo del estudio.

A todos los pacientes se les informó y registró su consentimiento para participar en el estudio.

\section{Variables}

La variable predictora del estudio es el uso de benzodiacepinas, y las variables resultado la glucosa preprandrial y la detección de hipoglucemias medidas en autocontrol. Otras variables medidas fueron: sexo, edad, tratamiento farmacológico con HGO y BZD, tipo y dosis. Se consideró concentración de glucosa normal según bibliografía $70-110 \mathrm{mg} / \mathrm{dl}$ y anormal $>110 \mathrm{mg} / \mathrm{dl}$ [2].

La muestra del estudio se dividió en dos grupos: pacientes con prescripción conjunta de $\mathrm{HGO}+\mathrm{BZD}$ o solo con HGO. A su vez a estos se los agrupó en función de la cifra de glucemias promedio preprandiales con valores dentro o fuera del rango esperado. Se analizó cada HGO y BZD de manera individual y se los relacionó entre sí.

\section{Análisis estadístico}

Para el análisis de los datos recogidos se utilizaron los programas informáticos Microsoft Excel ${ }^{\circledR}$ e Infostat ${ }^{\circledR}$ para examen estadístico.

Se planteó una prueba de hipótesis para muestras independientes entre los grupos considerando como hipótesis nula $\left(\mathrm{H}_{0}\right)$ considerando que no había diferencias entre el tratamiento de ambos grupos de pacientes. Se planteó como variable cuantitativa continua los niveles de glucemia medidos. Se utilizó la prueba $t$ de Student bilateral con 85 grados de libertad y un intervalo de confianza del 95\%.

Teniendo en cuenta que la hipótesis planteada orienta a observar si 
la prescripción de benzodiacepinas ayuda a la regulación de la glucemia, tendiendo a disminuirla, se analizó en cada paciente el nivel de glucemia más bajo que tuvo registrado y se lo correlacionó a su vez con el consumo de benzodiacepinas y un HGO específico, observando la presencia de hipoglucemias.

\section{Resultados}

De un total de 172 pacientes diabéticos que asistieron a la farmacia 87 cumplieron con los criterios de inclusión propuestos, 40 varones y 47 mujeres, con una edad media de 60 años. Solo 29 del total incluido en el estudio presentaron valores de glucemia preprandial dentro del rango.

De ellos, 43 pacientes tuvieron prescripción en forma concomitante de HGO y BZD, 19 hombres y 24 mujeres con una edad promedio de 59 años. De este grupo 21 pacientes presentaron glucemia en rango y 22 por encima del rango normal.

De los pacientes con tratamiento exclusivo de HGO, 22 hombres y 22 mujeres con una edad promedio de 58 años, solo 8 tuvieron niveles de glucemia en rango.

Si se compara los pacientes HGO + BZD frente a los que solo utilizaron HGO, se encuentra que la diferencia entre estos grupos es significativa $(\mathrm{p}=0,008)$ con un valor de t Student de 3,5. Los pacientes sin BZD tuvieron variaciones en los niveles de glucemia desde $134,9 \mathrm{mg} / \mathrm{dl}$ a $158,5 \mathrm{mg} / \mathrm{dl}$; mientras que los pacientes con HGO + BZD desde 108,5 mg/dl a 129,9 mg/ dl (figura 1).

Metformina fue el HGO que más se prescribió, en 64 (74\%) pacientes, ya sea combinado ( $\mathrm{n}=22$ pacientes) o como monoterapia $(n=42)$. La asociación con glimepirida fue la más utilizada con un total de 8 pacientes. Los HGO menos prescritos fueron acarbosa en 2 pacientes y rosiglitazona con 4 pacientes y 17 pacientes utilizaron sulfonilureas en monoterapia.

Las benzodiacepinas más prescritas fueron, en primer lugar, alprazolam con 15 pacientes, en dosis de $0,5 \mathrm{mg}$ (12 pacientes), $1 \mathrm{mg}$ (2 pacientes) y $0,25 \mathrm{mg}$ (1 paciente). En segundo lugar diazepam con prescripción en 13 pacientes, en dosis de 10 $\mathrm{mg}$ (2 pacientes), $5 \mathrm{mg}$ (8 pacientes), $2 \mathrm{mg}$ (3 pacientes).

El resto de las benzodiacepinas prescritas fueron bromazepam (7 pacientes), clonazepam (6 pacientes), siendo las de menor prescripción midazolam y lorazepam (1 paciente cada caso).

En la tabla 1 se describen los pacientes con HGO, BZD y sus relaciones.

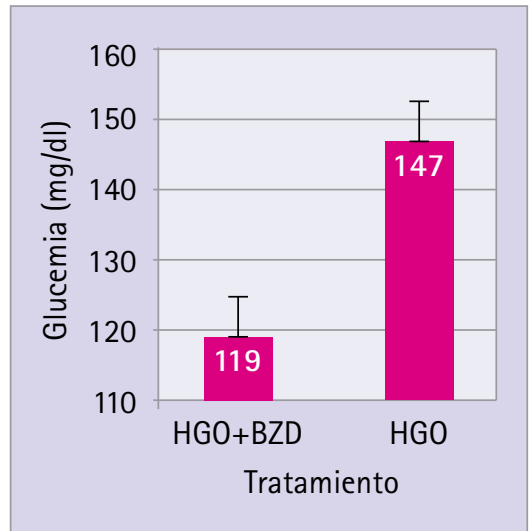

Figura 1 Rango de variación de la glucemia en ambos grupos

\section{Análisis de concentración}

de glucosa, relación con prescripción de benzodiacepinas e hipoglucemiantes orales en monoterapia

Los diabéticos que tuvieron prescripción de metformina en monoterapia fueron 42. De ellos, 20 pacientes prescripción conjunta con benzodiacepinas y 22 solo HGO. Según los valores de glucemia preprandial, del primer grupo se encontraron 7 que presentaron valores dentro del rango de glucemia, frente a 13 que tuvieron niveles fuera de rango (mayores).

Con respecto a los que no utilizaron benzodiacepinas, 5 pacientes tuvieron niveles de glucemia en rango y 17 presentaron glucemias fuera de

Tabla 1 Número de pacientes según HGO, BZD y sus relaciones

\begin{tabular}{|c|c|c|c|c|c|c|c|c|}
\hline \multirow{2}{*}{ HGO } & \multirow{2}{*}{$\begin{array}{l}\text { Metformina } \\
\text { (MET) }\end{array}$} & \multirow{2}{*}{ Sulfonilurea } & \multirow{2}{*}{ Rosiglitazona } & \multirow{2}{*}{ Acarbosa } & \multicolumn{4}{|c|}{ Asociaciones $(\mathrm{n}=22)$ Metfomina +} \\
\hline & & & & & Glimepirida & Gliclazida & Glibenclamida & Rosiglitazona \\
\hline $\begin{array}{l}\text { Pacientes } \\
(n=87)\end{array}$ & 42 & 17 & 4 & 2 & 8 & 6 & 7 & 1 \\
\hline $\begin{array}{l}\operatorname{Sin} B Z D \\
(n=44)\end{array}$ & 22 & 9 & 1 & 1 & 4 & 4 & 3 & -- \\
\hline $\begin{array}{l}\text { Alprazolam } \\
(n=15)\end{array}$ & 9 & $\begin{array}{l}1 \text { glimepirida } \\
1 \text { gliclazida }\end{array}$ & 2 & & & & 2 & \\
\hline $\begin{array}{l}\text { Diazepam } \\
(n=13)\end{array}$ & 5 & $\begin{array}{l}3 \text { glibenclamida } \\
2 \text { glimepirida }\end{array}$ & & 1 & 1 & 2 & & 1 \\
\hline $\begin{array}{l}\text { Bromazepam } \\
(n=7)\end{array}$ & 2 & 1 gliclazida & & & 2 & & 2 & \\
\hline $\begin{array}{l}\text { Clonazepam } \\
(n=6)\end{array}$ & 3 & 1 glimepirida & 1 & & 1 & & & \\
\hline Midazolam & 1 & & & & & & & \\
\hline Lorazepam & & 1 gliclazida & & & & & & \\
\hline BZD $(n=43)$ & 20 & 8 & 3 & 1 & 4 & 2 & 4 & 1 \\
\hline
\end{tabular}




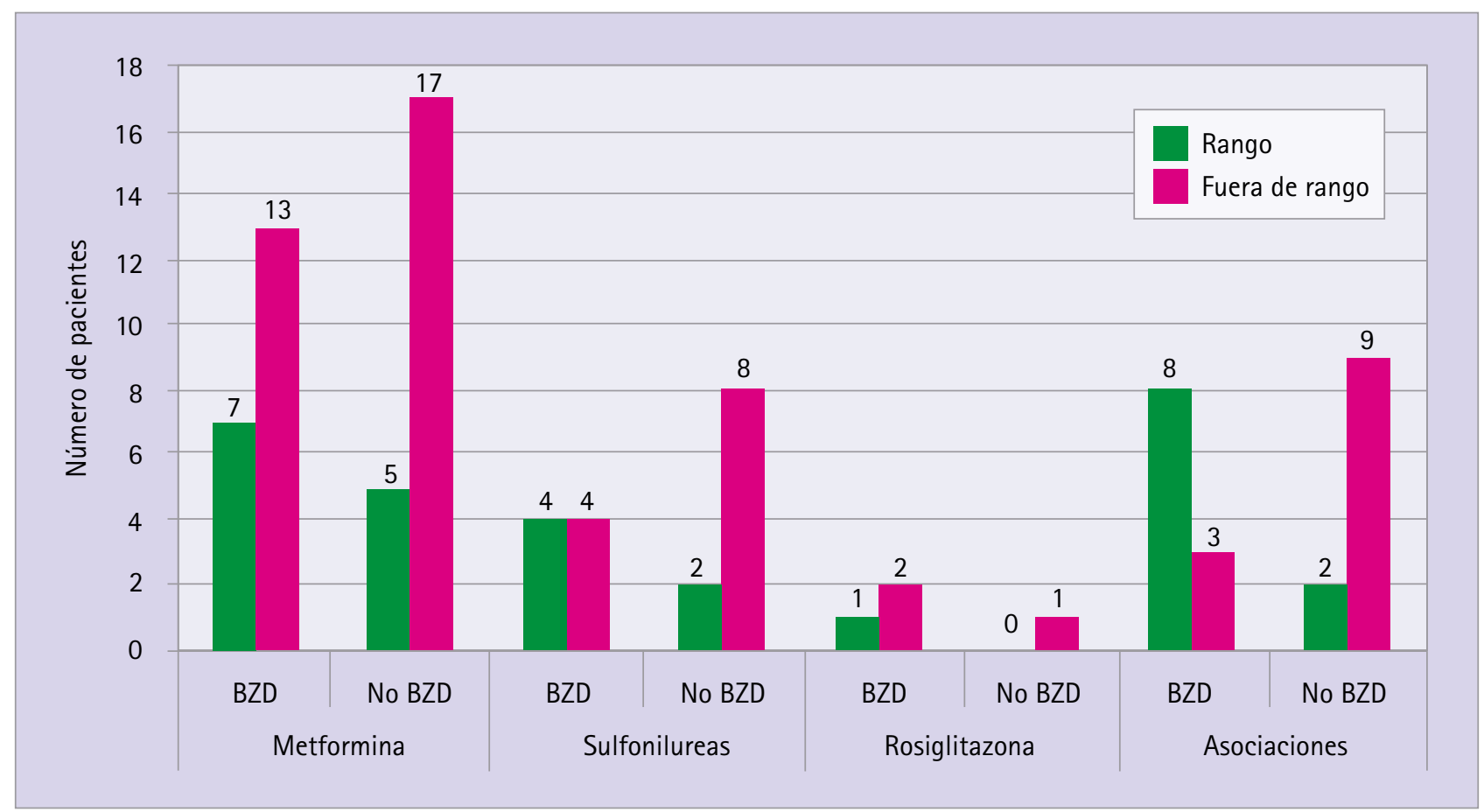

Figura 2 Cantidad de pacientes, prescripciones en monoterpia y valores de glucemias

rango. Para estos pacientes bajo prescripción de metformina, alprazolam fue la benzodiacepina más prescrita (9 pacientes).

De los 17 pacientes con sulfonilureas: glibenclamida (4), gliclazida (7) y glimepirida (6), 8 de ellos presentaron tratamiento combinado con benzodiacepinas. La benzodiacepina más prescrita en combinación con sulfonilureas fue diazepam, en 3 (35\%) pacientes.
De los 2 pacientes que tuvieron prescripción de acarbosa, uno tenía prescripción de benzodiacepina y el otro no. El paciente diabético que utilizó diazepam tuvo niveles de glucemia preprandial dentro del rango, mientras que en el otro paciente que solo tuvo prescripción de HGO sus glucemias estuvieron fuera de rango.

Se encontraron 4 pacientes con prescripción de glitazonas (rosiglitazona), de los cuales 3 tenían pres-

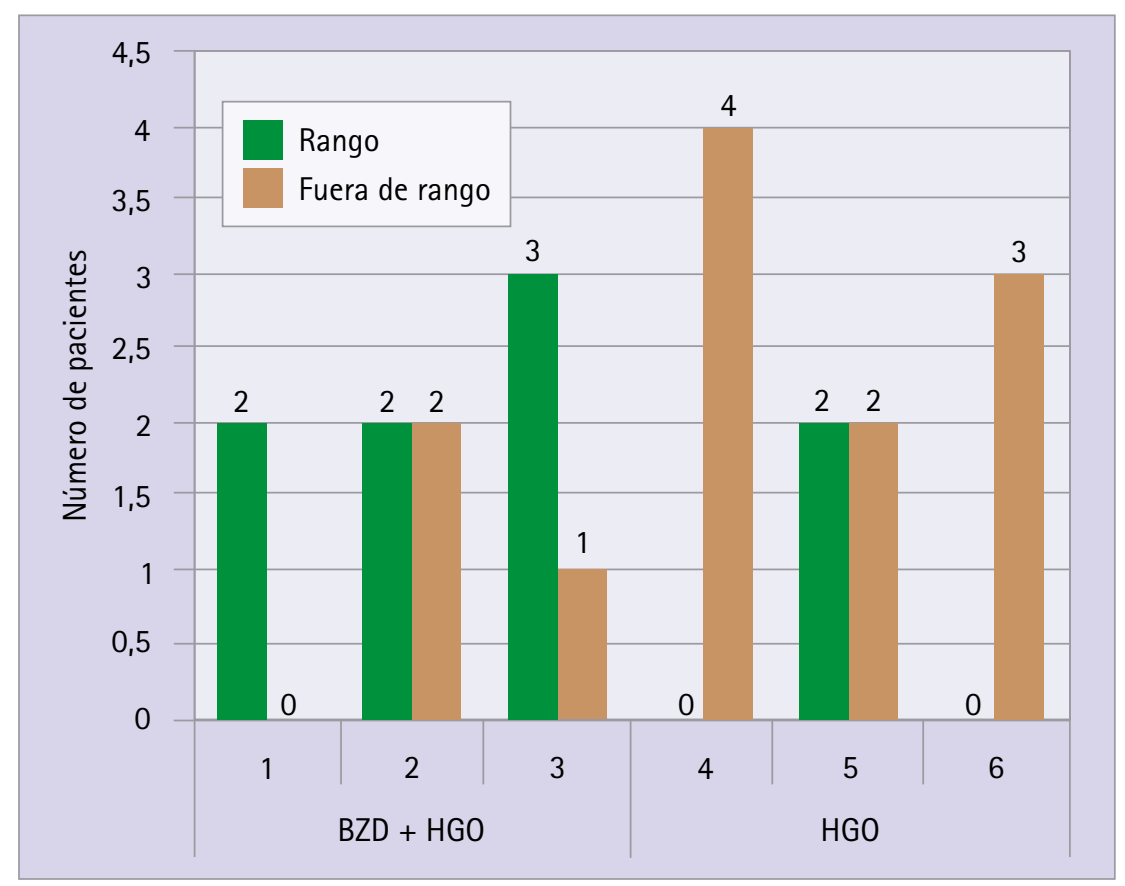

Figura 3 Número de pacientes, prescripciones en asociaciones de HGO con o sin uso de BZD y valores de glucemias

cripción de BZD (2 alprazolam y 1 clonazepam). Los valores de glucemia preprandial de estos 3 pacientes fueron por encima del rango para 2; al igual que el paciente que utilizó solo rosiglitazona. En la figura 2 se pueden observar los diferentes grupos de HGO y la cantidad de pacientes con valores dentro o fuera de rango de glucemias.

Análisis de concentración de glucosa, relación con prescripción de benzodiacepinas e hipoglucemiantes orales en asociaciones

22 pacientes recibieron tratamiento de HGO combinados, el 50\% tenía prescripción conjunta de benzodiacepinas. 8 pacientes presentaron niveles de glucemia preprandial en rango con benzodiacepinas; el resto presentó niveles por fuera. De los pacientes que sólo utilizaron HGO, 2 tuvieron glucemia preprandial en rango y 9 alterada. El análisis detallado por cada asociación se puede observar en la figura 3 (no se muestra paciente con rosiglitazona).

Las benzodiacepinas más prescritas junto con las asociaciones de HGO fueron diazepam (3 pacientes) y bromazepam (4 pacientes).

6 pacientes tuvieron tratamiento con gliclazida y metformina, 2 con prescripción de benzodiacepinas 
(ambos con diazepam) y glucemia preprandial en rango. Los 4 pacientes que solo emplearon asociación de HGO tuvieron valores de glucemia fuera de rango.

1 paciente tuvo tratamiento con rosiglitazona y metformina. El mismo empleó diazepam y obtuvo valores de glucemia preprandial dentro del rango esperado.

Los pacientes con tratamiento de glimepirida y metformina fueron 8, de los cuales 4 tuvieron prescripción de benzodiacepinas. El $50 \%$ de los pacientes de cada grupo (HGO con o sin benzodiacepina) tuvieron valores de glucemia preprandial en rango. Se prescribió en 2 pacientes bromazepam, diazepam y en solo 1 paciente alprazolam.

7 fueron tratados con glibenclamida y metformina. 4 pacientes utilizaron benzodiacepinas, de ellos, 3 tuvieron valores de glucemia preprandial en rango. Todos los pacientes que solo se trataron con los HGO tuvieron los niveles de glucemia fuera del rango. Las benzodiacepinas más prescritas fueron alprazolam y bromazepam (2 en ambos casos).

Análisis de glucemias preprandiales en pacientes en relación a las benzodiacepinas más prescritas

En los 15 pacientes que emplearon alprazolam y los 13 con diazepam la metformina fue el HGO más prescrito, incluidos los 7 pacientes que emplearon bromazepam, en monoterapia o combinaciones de HGO. 6 pacientes usaron clonzepam midazolam, 1 paciente con metformina y glucemia en rango, y lorazepam 1 paciente con gliclazida y glucemia fuera del rango.

Si se realiza el análisis de glucemias en función de BZD se encuentra que para alprazolam y clonazepam predominaron los valores por encima del rango de glucemia preprandial, mientras que para diazepam y clonacepam fueron mayoría los pacientes con glucemias preprandiales normales (figura 4).

\section{Riesgo de hipoglucemias}

De los 87 pacientes del estudio se encontraron 28 con valores de glucemia menores de $70 \mathrm{mg} / \mathrm{dl}$. De ellos, 15 tuvieron prescripción de BZD y

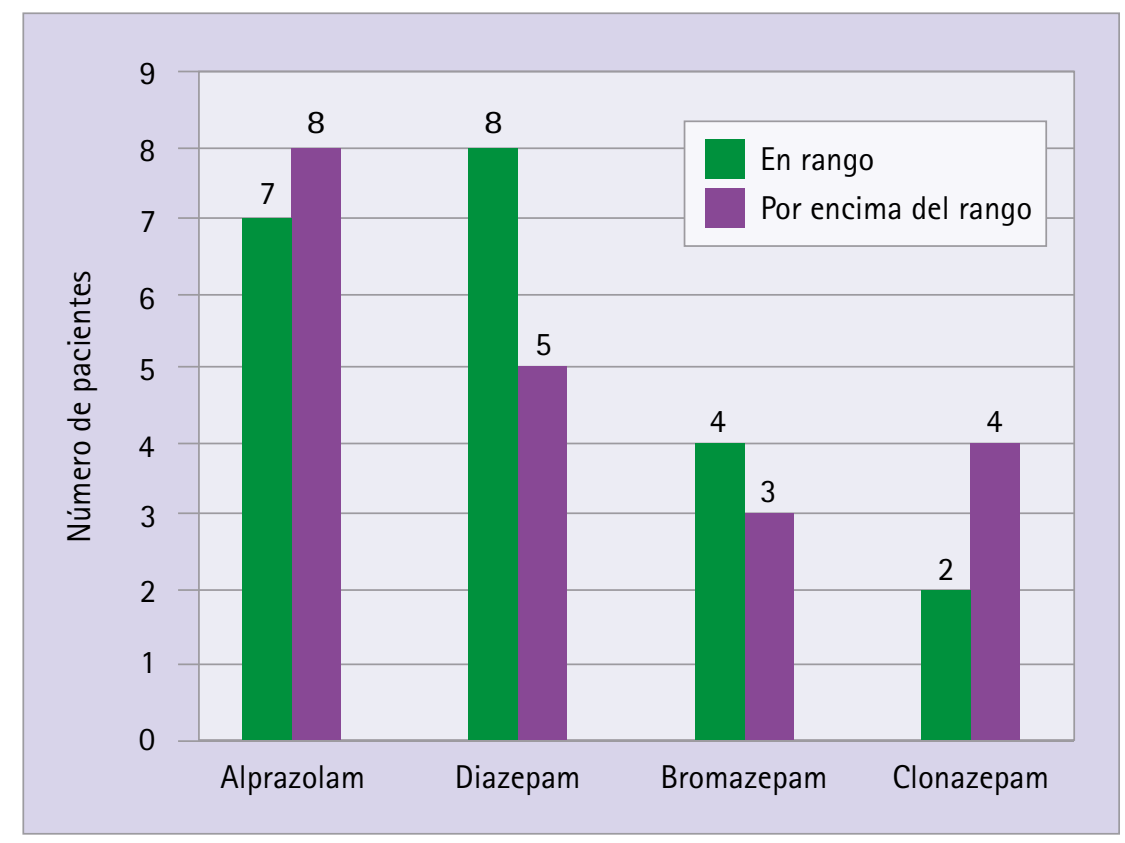

Figura 4 Relación entre pacientes con prescripción de BZD y glucemias

13 sólo HGO. Es decir, que de los 43 diabéticos totales que fueron tratados con benzodiacepinas, 15 (35\%) presentaron hipoglucemia.

\section{Discusión}

Los estudios que se han realizado hasta el momento sobre la relación entre benzodiacepinas, diabéticos tipo 2 e HGO no son frecuentes ni recientes. En 1995, Lutsman y col publicaron datos sobre la acción de alprazolam en pacientes diabéticos. El mismo explica los efectos del alprazolam en la regulación de la glucemia en diabéticos, mediante un ensayo a doble ciego, en el cual se llevaron a cabo análisis a pacientes con la patología y se determinó que su consumo, a corto plazo, logra una mejor regulación de la glucosa. Este tratamiento en pacientes ansiosos con mal control de la diabetes resultó en una disminución de ansiedad, respectiva, y mejora la regulación de la glucemia. El mecanismo planteado implica que alprazolam opera como amortiguador de la respuesta neurohormonal al estrés, obteniendo como resultado controlar los niveles de glucemia. Esta benzodiacepina modificaría el efecto hiperglucémico asociado al estrés y aumentaría significativamente el nivel de insulina plasmática [8].

En nuestro estudio se encontraron, en los pacientes con prescripción de alprazoplam, niveles de glucemia alterados en el 53\%, no pudiéndose afirmar que el uso conjunto se relacione con esto a diferencia de lo propuesto por Lustman. Estos resultados pueden estar relacionados con la situación personal de cada paciente estudiado, el tipo de alimentación, estado anímico, cumplimiento terapéutico, etc.

Gómez y Barros plantearon una teoria contraria a la anterior: clonazepam aumenta los niveles de glucemia normales en ratas diabéticas [9]. Si bien en este grupo de pacientes con prescripción de clonazepam se observaron valores fuera de rango de glucemia preprandial no podemos establecer ninguna afirmación debido a que el número de pacientes con esta prescripción [6 pacientes) fue pequeño.

Con respecto a diazepam, Garabadu y Krishnamurthy sostuvieron que esta benzodiacepina potenciaba el efecto antidiabético de la metformina, enfocándose puntualmente en situaciones de estrés. De este modo, concluyeron que la combinación de ambos atenuaba significativamente la hiperglucemia y además se observaban otros beneficios como mejoría de la función mitocondrial, integridad y estrés oxidativo en el hipocampo, hipotálamo, corteza prefrontal, cuerpo estriado, amígdala y núcleo accumbens. Es decir, la prescripción conjunta de metformina asociada con diazepam podría ser una mejor opción terapéutica en el tratamiento de la DM2 bajo condición de estrés [10]. En lo que respecta a nuestro estudio, 
los pacientes que presentaron prescripción de metformina y a su vez de diazepam tuvieron niveles de glucemia preprandiales con predominio de los valores normales.

En los diabéticos tipo 2 que tuvieron prescripción de diazepam predominaron los niveles de glucemia preprandial y postprandial normales, lo que significa una buena regulación de la glucemia con esta benzodiacepina.

El análisis estadístico solo se llevó a cabo sobre los valores de glucemia preprandial, debido a que los niveles postprandiales no pueden ser analizados en este trabajo por el diseño metodológico del mismo debido a la existencia de una variable cualitativa que no se puede medir como la dieta del paciente. Se desconocía el tipo de alimento que los pacientes ingirieron antes de realizar la medición. Esta variable otorga gran influencia sobre los valores de glucemia obtenidos; por ello solo se desarrolló el análisis de la glucemia preprandial exclusivamente.

Teniendo en cuenta que determinados HGO, como glibenclamida, presentan como efectos adversos hipoglucemia, en este estudio identificamos que 7 de 11 presentaron glucemias menores a $70 \mathrm{mg} / \mathrm{dl}$, siendo 3 los que estuvieron tratados con benzodiacepinas. Esto llevaría a considerar como criterio de alerta el uso de este HGO en conjunto con BZD.

En este estudio diazepam ha sido la benzodiacepina que logró un mejor control de la glucemia; ello puede deberse a que este fármaco presenta una vida media mayor a 30 horas, lo que contribuiría a lograr estabilizar la glucemia en mayor proporción. El resto de las benzodiacepinas empleadas no lograron un control semejante.

Se consideró limitante en la metodología propuesta que, si bien se realizó un criterio de selección en la cantidad de auto mediciones realizadas por los pacientes, no se estableció el método de medición en función del aparato utilizado ni el tiempo en que el paciente realiza esta determina- ción. Igualmente se considera como una posible limitación del estudio, el distinto efecto de la asociación de los diferentes tipos de hipoglucemiante y de benzodiacepinas, ya que en el análisis bivariante realizado en el estudio no se ha contemplado un subanálisis en función de estas variables.

Si bien sería preferible para tener un conocimiento del control de la glucemia real de estos pacientes la determinación de hemoglobina glicosilada, por tratarse de pacientes que retiran sus medicamentos en una farmacia comunitaria, solo se dispone de los datos de autocontrol que son obtenidos de sus aparatos al retirar los insumos y medicamentos mensuales. Sin embargo, no se descarta que en el futuro se pueda solicitar esta información a los pacientes para tener un perfil de la evolución del tratamiento utilizado.

Para concluir, se observó que la prescripción de benzodiacepinas junto con HGO en este grupo de pacientes diabéticos tipo 2 tiene como consecuencia un descenso mayor de los niveles de glucemia preprandial con posibilidad de aparición de hipoglucemias que en aquellos que sólo emplearon monoterapia de HGO. Sin embargo, debido a las limitaciones metodológicas explicadas previamente no se puede concluir una relación directa. Queda por establecer en futuras investigaciones los posibles mecanismos de acción de estos tratamientos prescritos en forma conjunta.

\section{Agradecimientos}

A la farmacéutica María L. Tiscornia, que fue codirectora del trabajo final de carrera.

\section{Referencias bibliográficas}

1. Pereira Despaigne OL, Palay Despaigne MS, Rodríguez Cascaret A, Neyra Barros RM, Chia Mena MA. Hemoglobina glucosilada en pacientes con diabetes mellitus. MEDISAN [Internet]. 2015; 19(4):555-561. [Acceso 13/11/17]. Disponible en: http://scielo.sld.cu/ scielo.php?script=sci_arttextEtpi-
$d=S 1029-30192015000400012 \& \ln -$ $\mathrm{g}=\mathrm{es}$

2. Lorenzo P, Moreno A, Lizasoain I, Leza JC, Moro MA, Portolés A. Velázquez Farmacología Básica y Clínica. Madrid, España: Editorial Médica $\mathrm{Pa}-$ namericana; 2008. Pág. 1375.

3. Organización Mundial de la Salud OMS. La diabetes una enfermedad crónica [Internet]. 2016. Ginebra, Suiza. [Acceso 13/11/17]. Disponible en: http://www.who.int/diabetes/es/

4. Collins R, Armitage J, Parish S, Sleigh P, Peto R. Study of colesterol-lowering with symvastatin in 5963 people with diabetes: a randomize placebo-controlled trial. Health Protection 2008; 361:205-216.

5. Asociación Americana de Diabetes, ADA. Análisis de cetonas [Internet]. 2016. Alexandría, Estados Unidos. [Acceso 9/11/17]. Disponible en: http:// www.diabetes.org/es/vivir-con-diabetes/tratamiento-y-cuidado/el-control-de-la-glucosa-en-la-sangre/anlisis-de-cetonas.html

6. Ministerio de la Salud de la Nación. ¿Qué son las enfermedades no transmisibles? [Internet]. 2016. Buenos Aires, Argentina. [Acceso 23/10/17]. Disponible en: http://www.msal.gob. ar/ent/index.php/informacion-para-ciudadanos/ique-son-icuales-son

7. Sandoval-Salazar C, Ramírez-Emiliano J, Solís-Ortiz S El sistema de inhibición GABAérgico implicado en la regulación de la ingesta alimentaria y obesidad. Rev Mex Neuroci. 2013; 14 (5):262-271.

8. Lustman PJ, Griffith LS, Clouse RE, Freedland K, Eisen S, Rubin E, et al. Effects of alprazolam on glucose regulation in diabetes: results of double-blind, placebo-controlled trial. Diabetes Care 1995; 18:1133-1139. doi:10.2337/diacare.18.8.1133

9. Gómez R, Barros HM. Clonazepam increases in vivo striatal extracellular glucose in diabetic rats after glucose overload. Pharmacol Biochem Behav. 2003; 76: 440-450. doi:10.1016/j.pbb. 2003.08.018

10. Garabadu D, Krishnamurthy S. Diazepam Potentiates the Antidiabetic, Antistress and Anxiolytic Activities of Metformin in Type-2 Diabetes Mellitus with Cooccurring Stress in Experimental Animals. Biomed Res Int. 2014, ID: 693074, 15 pages. doi:10.1155/2014/693074 